\title{
M1 Protein-Dependent Intracellular Trafficking Promotes Persistence and Replication of Streptococcus pyogenes in Macrophages
}

\author{
Erika Hertzén $^{a} \quad$ Linda Johansson $^{a}$ Robert Wallin ${ }^{a}$ Heike Schmidt ${ }^{a}$ Mirko Krolla \\ Anders P. Rehn ${ }^{a}$ Malak Kotb ${ }^{c}$ Matthias Mörgelin ${ }^{b} \quad$ Anna Norrby-Teglund ${ }^{a}$ \\ ${ }^{a}$ Center for Infectious Medicine, Karolinska Institutet, Stockholm, and bepartment of Clinical Science, BMC, \\ Lund University, Lund, Sweden; ' Department of Molecular Genetics, Biochemistry and Microbiology, \\ University of Cincinnati, Cincinnati, Ohio, USA
}

\section{Key Words}

Streptococcus pyogenes Intracellular persistence .

Macrophage $\cdot$ M protein $\cdot$ Host-pathogen interaction

\begin{abstract}
Streptococcus pyogenes is an important human pathogen that causes a variety of diseases including life-threatening invasive diseases, such as toxic shock and deep tissue infections. Although S. pyogenes are classically considered extracellular pathogens, a clinical significance of an intracellular source has been emphasized. In patients with deep tissue infections, an intracellular reservoir of $S$. pyogenes within macrophages was shown to contribute to prolonged bacterial persistence. Here we demonstrate that intracellular survival of S. pyogenes in macrophages is associated with an M1 protein-dependent intracellular trafficking in the phagosomal-lysosomal pathway, which results in impaired fusion with lysosomes. The phagocytic vacuoles harbouring M1 protein-expressing bacteria not only served as a safe haven for the bacteria, but also as a replicating niche. An M1 protein-dependent modulation of macrophages was further supported by differences in NF- $\mathrm{kB}$ signalling between cells infected with either the wild-type or M1 protein-deficient strains, thereby indicating a suppressed inflammatory re-
\end{abstract}

sponse when M1 protein was involved. Evidence of egress of bacteria out of their host cell and subsequent re-infection of new cells emphasize the importance of intracellular bacteria as a reservoir for dissemination of infection and continued tissue injury.

Copyright $\odot 2010$ S. Karger AG, Basel

\section{Introduction}

Streptococcus pyogenes are important human pathogens, listed number 9 on the list of 'global killer pathogens' with an estimated 500,000 deaths yearly [1]. It causes a variety of diseases ranging from mild uncomplicated skin and throat infections to severe invasive diseases and post-streptococcal sequelae. Two of the most severe invasive manifestations include streptococcal toxic shock syndrome and necrotizing fasciitis [1], which are hyperinflammatory rapidly progressing conditions associated with high mortality and morbidity despite adequate antibiotic therapy in most cases. Superantigens and the M1 protein are recognized as central triggers of hyper-inflammatory responses and vascular leakage, which consequently results in the systemic toxicity associated with these infections [2-4]. In addition, S. pyogenes is equipped

\section{KARGER}

Fax +41613061234 E-Mail karger@karger.ch www.karger.com

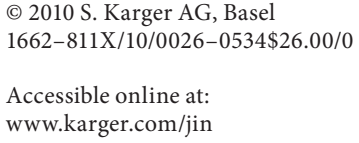

Dr. Anna Norrby-Teglund

Center for Infectious Medicine F59

Karolinska Institutet

SE-141 86 Stockholm (Sweden)

Tel. +4685858 7296, Fax +468746 7637, E-Mail anna.norrby-teglund@ki.se 
with an impressive array of virulence factors that promote colonization and persistence through various immunomodulatory activities ranging from proteolytic cleavage of immune effector proteins to avoidance of phagocytic killing [reviewed in 5].

There have been reports that $S$. pyogenes, which is classically known as an extracellular pathogen, may invade and reside intracellularly in host cells. Intracellular Streptococci have been demonstrated in vitro in epithelial cells $[6,7]$ and in neutrophils $[8,9]$. Furthermore, an intracellular reservoir of $S$. pyogenes, residing predominantly within macrophages, was demonstrated in patients with acute deep tissue infections in patients [10]. The fact that many of these patients had persistent massive bacterial load despite intravenous antibiotic therapy for a prolonged time underscored a major clinical problem with antibiotic eradication failure at the tissue site of infection.

Normally bacteria are killed by the conventional phagosomal-lysosomal degradation pathway following phagocytosis. However, intracellular pathogens have acquired specific mechanisms to avoid this default pathway and promote their survival in phagocytic cells $[11,12]$. The 4 major evasion strategies involve (1) avoidance of fusion between endocytic vacuole and lysosomes, (2) modulation of phagosome biogenesis, (3) adaptation to the acidic environment in the phagolysosome and (4) escape from the phagolysosome into the cytoplasm. In macrophages, this may be achieved by bacteria-induced modulation of macrophages into the anti-inflammatory M2 phenotype, in contrast to the pro-inflammatory M1 phenotype usually associated with infection [13]. The two phenotypes may be characterized by their cytokine/chemokine receptor profiles, and their polarization was recently shown to be orchestrated by NF- $\mathrm{KB}$ signalling [14]. In $S$. pyogenes infections, various intracellular survival strategies have been proposed including caveolae-mediated uptake in epithelial and endothelial cells [7], streptococcal M1 protein-mediated inhibition of granule fusion with the phagosomes in neutrophils [9] and streptolysin $\mathrm{O}$-mediated avoidance of lysosomal degradation in epithelial cells [15].

In this study, we have explored the intracellular survival strategies of $S$. pyogenes in macrophages, as this is the principal reservoir during acute tissue infections [10]. Our findings demonstrate that intracellular survival is associated with an M1 protein-dependent intracellular trafficking in the phagosomal-lysosomal pathway, which results in impaired fusion with lysosomal vesicles. The phagocytic vacuoles harbouring M1 protein-expressing bacteria not only served as a safe haven for the bacteria, but also as a replicating niche. Further studies emphasized the importance of this intracellular bacterial source as a reservoir for dissemination of infection and continued tissue injury.

\section{Material and Methods}

\section{Ethics Statement}

The Human Subjects Review Committee of Karolinska Institutet approved the studies using blood obtained from healthy volunteers. All blood donors were anonymous and therefore written informed consent was not required.

Primary Human Cell Preparation and Culture Conditions

Human monocyte-derived macrophages (hMDMs) were prepared essentially as previously decribed [10]. Briefly, for confocal and electron microscopy assays, peripheral blood mononuclear cells were isolated from whole blood of healthy donors through density gradient centrifugation using Lymphoprep (Axis-Shield). Monocytes were enriched through plastic adherence for $2 \mathrm{~h}$ in serum-free media in 12- or 24-well plates (Corning) with or without coverslips and allowed to differentiate into macrophages in RPMI 1640 supplemented with 10\% FCS for 7 days. For flow cytometry, cytotoxicity and Western blot assays, monocytes were isolated using Rosette Sep (Stemcell Technologies) separation followed by a Lymphoprep (Axis-Shield) centrifugation step. Monocytes were then seeded in 6-well low-adherence plates (Corning) in RPMI containing $10 \%$ FCS and $50 \mathrm{ng} / \mathrm{ml}$ macrophage colonystimulating factor (Immunotools), and cultured for 5-7 days to obtain hMDMs. Maturation of the cells was assessed morphologically using light microscopy and by flow cytometry studying MHC II, CD68 and CD14 markers.

\section{Bacterial Strains}

A clinical M1T1 isolate of S. pyogenes (strain 5448) [16] and its mutant lacking the $\mathrm{M} 1$ protein $(5448 \Delta \mathrm{M} 1)$ were grown at $37^{\circ} \mathrm{C}$ to stationary phase in Todd-Hewitt broth supplemented with $1.5 \%$ yeast extract and diluted to the required inoculum, 10-40 colonyforming units (CFU)/cell depending on experimental assay. A green fluorescent protein (GFP) expressing 5448 strain was created by use of the plasmid pGFP1 [17]. Streptococcal transformants were generated by electroporation essentially as described previously [18], and selected on blood agar plates containing erythromycin $(100 \mu \mathrm{g} / \mathrm{ml})$. GFP-expressing 5448 strains were cultured as above using Todd-Hewitt supplemented with $150 \mu \mathrm{g} /$ $\mathrm{ml}$ erythromycin. Erythromycin-supplemented cell culture media was always used in infections with GFP-expressing strains. Escherichia coli DH5a containing the plasmid pGFP1 was grown at $37^{\circ} \mathrm{C}$ in Luria broth containing $85 \mu \mathrm{g} / \mathrm{ml}$ chloramphenicol.

In vitro Infection Assay

S. pyogenes strains were co-cultured with hMDMs at $37^{\circ} \mathrm{C}$ and $5 \% \mathrm{CO}_{2}$ for different time points. A multiplicity of infection (MOI) of 20-30 CFU/cell was used for early time points 5-60 min, whereas an MOI of $10 \mathrm{CFU} /$ cell was used for later time points 2$12 \mathrm{~h}$, unless otherwise specified. At $2 \mathrm{~h}$ after infections, extracellular and adherent bacteria were killed by addition of $125 \mu \mathrm{g} / \mathrm{ml}$ 
gentamicin and $2.5 \mu \mathrm{g} / \mathrm{ml}$ penicillin $\mathrm{G}$ for $1 \mathrm{~h}$, followed by washing in PBS twice, and addition of RPMI 1640 supplemented with $1 \mu \mathrm{g} / \mathrm{ml}$ penicillin $\mathrm{G}$ throughout the rest of the experiment. Egress of bacteria out of cells was assessed in culture media where the antibiotic had been removed after the initial killing of extracellular and adherent bacteria. CFU in cell culture media were enumerated through plating on blood agar plates at different time points post antibiotic removal.

\section{Immunofluorescent Staining}

hMDMs on coverslips were fixed in $1 \%$ freshly prepared paraformaldehyde for $30 \mathrm{~min}$. Intracellular staining was done essentially as previously described [10]. Briefly, cells were blocked by $2 \%$ FCS, followed by avidin and biotin blocking (Vector Laboratories). Primary antibodies included polyclonal antiserum against Lancefield group A carbohydrate (Difco), antiEEA-1 (N-19) and anti-LAMP-1 (H5G11) (both from Santa Cruz Biotechnology). Further blocking was achieved by $1 \%$ normal goat serum or $1 \%$ normal rabbit serum depending on the species of the secondary antibody. This was followed by incubation with a secondary biotinylated antibody, whereafter a streptavidinconjugated fluorophor (Alexa Fluor 594 or Alexa Fluor 488; Molecular Probes) was added. All dilutions and washing steps were done in $0.1 \%$ PBS-saponin. The coverslips were mounted with ProLong Gold mounting media with DAPI (Molecular Probes). Analysis was done using a Leica confocal scanner TCS SP II coupled to a Leica DMR microscope. For assessment of LysoTracker Red DND-99 (Molecular Probes), cells were incubated with $2.5 \mu \mathrm{M}$ of the solution together with GFP-expressing bacteria for $2 \mathrm{~h}$, after which the cells were washed and visualized using a Delta Vision spectris, wide-field microscope (Applied Precision) after $2-5 \mathrm{~h}$ of infection. Viability of intracellular bacteria was studied using the Baclight bacterial viability kit (Molecular Probes) as described by the manufacturer and analysed by a Leica DMR immunofluorescent microscope. Actin filaments were visualized by incubating the cells with Alexa Fluor 555 phalloidin (Molecular Probes) as described by the manufacturer and analysed in a Delta Vision spectris, wide-field microscope (Applied Precision).

\section{Electron Microscopy}

hMDMs were fixed in $2.5 \%$ glutaraldehyde in $0.15 \mathrm{M}$ sodium cacodylate $\mathrm{pH} 7.4$ over night at room temperature. They were subsequently washed with $0.15 \mathrm{M}$ sodium cacodylate and post-fixed with $1 \%$ osmium tetroxide for $2 \mathrm{~h}$ at $4^{\circ} \mathrm{C}$. This was followed by dehydration through a graded series of ethanol and embedding in Epon 812. The samples were sectioned using a LKB ultramicrotome and collected on grids followed by staining with uranyl acetate and lead citrate. Sections were analysed using an JEOL JEM 1230 electron microscope operated at $80 \mathrm{kV}$ accelerating voltage, and images were recorded with a Gatan Multiscan 791 CCD camera.

\section{Flow Cytometry}

$1 \times 10^{5}$ hMDMs were pre-incubated with or without Cytochalasin D (Sigma) $10 \mu \mathrm{g} / \mathrm{ml}$ for $30 \mathrm{~min}$ following addition of GFP expressing $S$. pyogenes at an MOI of $5 \mathrm{CFU} /$ cell for $2 \mathrm{~h}$. Then cells were fixed, processed with FACSCalibur (Becton Dickinson) and analysed with FlowJo software (TreeStar Inc.).
Western Blot Assay

$1 \times 10^{6} \mathrm{hMDM}$ were infected with 5448 or $5448 \Delta \mathrm{M} 1$ at an MOI of 5 CFU/cell or stimulated with LPS $100 \mathrm{ng} / \mathrm{ml}$ (Sigma) for $10 \mathrm{~min}$. Cells were lysed in Laemmeli sample buffer (Bio-Rad) and MilliQ water 1:1. Samples were resolved on 10-20\% gradient Trisglycine gels (Invitrogen) and then transferred to a polyvinylidene fluoride membrane (Invitrogen). Membranes were blocked with 5\% BSA (Sigma) and probed with the following antibodies: antiphospho-IкB $\alpha$, anti-phospho-p65 (Cell Signalling Technologies) and anti- $\beta$-actin (Sigma). Secondary antibodies included peroxidase-conjugated anti-mouse and peroxidase-conjugated antirabbit (Jackson ImmunoResearch). The proteins were visualized by Extended Duration Substrate kit (Thermoscientific) and a Fujifilm LAS-4000 Mini instrument. Optical density was assessed by Image J software $(\mathrm{NIH})$.

\section{Real-Time PCR}

hMDMs were infected in duplicate wells from 6 separate donors with either strains 5448 or $5448 \Delta \mathrm{M} 1$, or mock-infected for $1 \mathrm{~h}$, after which RNA was isolated using the RiboPure RNA Extraction Kit (Ambion). Concentration was measured using NanoDrop ${ }^{\circledR}$ spectrophotometer (NanoDrop Technologies). RNA was subsequently converted to cDNA with High Capacity cDNA Reverse Transcription Kit (Applied Biosystems). Fifty nanograms of reverse-transcribed RNA was used for qPCR analysis using primer-probe (FAM-MGB) gene expression assays from Applied Biosystems [18S (4352930), TNF $\alpha$ (Hs00174128_m1)]. qPCR reaction was performed in the 7500 Fast system using $2 \times$ Gene Expression Master Mix (both from Applied Biosystems) and in accordance with provided protocols. Results from well duplicates were pooled and analysed using the $\Delta \Delta \mathrm{Ct}$ method with mock-infected samples as calibrator and $18 \mathrm{~S}$ as endogenous control in MS Excel. Statistical analysis (paired t test) was performed in Graphpad Prism (Graphpad Software Inc.).

\section{Lactate Dehydrogenase Assay}

One day prior to the assay, hMDMs were harvested from 6 -well plates and $2.5 \times 10^{5}$ cells were seeded per well in a 48 -well plate in cell culture media lacking phenol-red, antibiotics and containing $2 \%$ FCS. At the day of infection, bacteria were added to the plates at an MOI of $5 \mathrm{CFU} /$ cell. After $2 \mathrm{~h}$ of infection, $1 \mu \mathrm{g} /$ $\mathrm{ml}$ penicillin $\mathrm{G}$ was added to kill off extracellular bacteria. Cell culture supernatants were taken at 6,9 and $12 \mathrm{~h}$ after infection. The amount of lactate dehydrogenase (LDH) in the cell culture media was measured using the CytoTox 96 cytotoxicity assay (Promega) according to the manufacturer's instructions. Briefly, $50 \mu \mathrm{l}$ of supernatant was mixed with $50 \mu \mathrm{l}$ of substrate reagent and incubated for $30 \mathrm{~min}$ at room temperature. Absorbance was measured at $490 \mathrm{~nm}$. Cytotoxicity was calculated as: [(sample $\mathrm{LDH}$ release - spontaneous $\mathrm{LDH}$ release)/(maximum LDH release - spontaneous LDH release)] $\times 100$. The spontaneous release equals the amount of LDH released by uninfected cells and the maximum released is the amount of $\mathrm{LDH}$ released by uninfected cells after incubation with $0.1 \%$ Triton X-100.

\section{Re-Infection Assay}

$1 \times 10^{5} \mathrm{hMDMs}$ were seeded per well in a 24 -well plate. Cells were infected with GFP-expressing 5448 for $1 \mathrm{~h}$ with an MOI of $40 \mathrm{CFU} / \mathrm{cell}$. Extracellular or adherent bacteria were killed off by antibiotics as described above, after which antibiotic-free media 
was added. Cells stained with FarRed (Molecular Probes), according to the manufacturer's instructions, were added and co-incubated with the infected cells. At different time points, all cells were collected and the percentage of FarRed/GFP double-positive cells was measured by flow cytometry on FACSCalibur (Becton Dickinson) and analysed with FlowJo software (TreeStar Inc.). Microscopical analyses of the infected cells were performed to confirm intracellular localization of $S$. pyogenes in the FarRed-stained cells.

\section{Statistical Evaluation}

Data were analysed by GraphPad Prism version 4.0 for Windows (Graphpad Software). Comparisons between groups were performed using a Mann-Whitney test, Wilcoxon signed rank test or paired t test. Differences were considered significant when $\mathrm{p}<$ 0.05 .

\section{Results}

\section{S. pyogenes Uptake in Macrophages Is Actin}

Dependent and Involves Long Asymmetrical

\section{Pseudopod Loops}

To gain insight into the uptake process, primary hMDMs were infected with a clinical M1T1 S. pyogenes isolate (5448) for defined time points, ranging from $5 \mathrm{~min}$ to several hours, and analysed at the ultrastructural level. Transmission electron microscopy analyses revealed bacterial uptake already after $5 \mathrm{~min}$ of infection. There were no apparent morphological differences with respect to membrane ruffles in infected or non-infected macrophages (fig. 1a-c). Ultrastructural data demonstrated spacious asymmetrical pseudopod loops surrounding the bacteria (fig. 1a) as well as bacteria tightly attached to the cell surface and encircled by membrane ruffle-like structures (fig. 1b). Taken together this resembles the process of triggered phagocytosis and coiling phagocy tosis. In contrast, no evidence of involvement of phagocytic cups or caveolae invaginations was found at the $S$. pyogenes membrane attachment sites. Furthermore, the uptake process was not dependent on secreted bacterial factors, as evident by the identical uptake of paraformaldehyde-fixed bacteria (data not shown).

As both phagocytosis and macropinocytosis are actindependent processes, we assessed the effect of inhibiting actin-polymerization by pre-treatment of cells with cytochalasin D. This resulted in a significant reduction in $S$. pyogenes uptake as compared to untreated cells $(\mathrm{p}=$ 0.0001 ) (fig. 1d). This was also supported by immunofluorescent stainings of infected cells, which revealed actin surrounding the bacteria at the process of entry (fig. 1e). Although some bacteria may induce their uptake by stimulating macropinocytosis, the long chains of $S$. pyogenes is likely a limitation for this uptake process. Furthermore, we did not observe any general stimulation of macropinocytic uptake of fluorescent dextran following $S$. pyogenes infection of macrophages (data not shown).

S. pyogenes Reside within Phagocytic Vacuoles, which Avoid Phagolysosomal Maturation

Transmission electron microscopy revealed that once inside the cells, $S$. pyogenes reside within spacious phagocytic vacuoles surrounded by an electron-dense membrane (fig. 2a). Throughout the experiments (up to $12 \mathrm{~h}$ ), intracellular bacteria remained within these vacuoles and no free bacteria could be detected in the cytosol of the cells. Next we explored the intracellular trafficking by mapping bacterial localization within defined endosomal compartments. For this purpose, a GFP-expressing 5448 isolate was constructed and used in the infection model. Immunostaining of infected cells and analyses by confocal microscopy demonstrated that the bacteria transiently co-localized with the early endosomal marker EEA-1 up to 15 min after infection (fig. 2b), after which the association was lost (data not shown). In contrast, no association of bacteria and the late endosomes/lysosomes could be demonstrated using either the lysosome-associated membrane protein 1 (LAMP-1) or the acidotropic stain LysoTracker that targets lysosomes (fig. 2c, d and fig. 3f, respectively). To rule out that the lack of association was due to an inactivation of GFP expression resulting from the acidic environment, both phase-contrast images and, most importantly, the DAPI channels were carefully screened for coccal chains and assessed in relation to GFP positivity. The data of DAPI-stained coccal structures were fully congruent with the GFP-positive bacteria, thus, supporting a lack of co-localization between wild-type 5448 bacteria and the lysosomes. Fixed bacteria behaved in a similar manner and did not associate with lysosomal markers (data not shown).

\section{Intracellular Survival of S. pyogenes in Macrophages \\ Is Dependent on M1 Protein}

Next we sought to identify bacterial factors that contribute to intracellular survival. Our principal candidate was the streptococcal M1 protein, as previous findings in neutrophils had implicated a role for this protein in impaired granule fusion [19]. For this purpose, a 5448 strain deficient in the M1 protein $(5448 \Delta \mathrm{M} 1)$ was used in the infection model. No evident morphological difference in the uptake process of the $5448 \Delta \mathrm{M} 1$ strain could be observed using electron-microscopic analysis of infected 
a
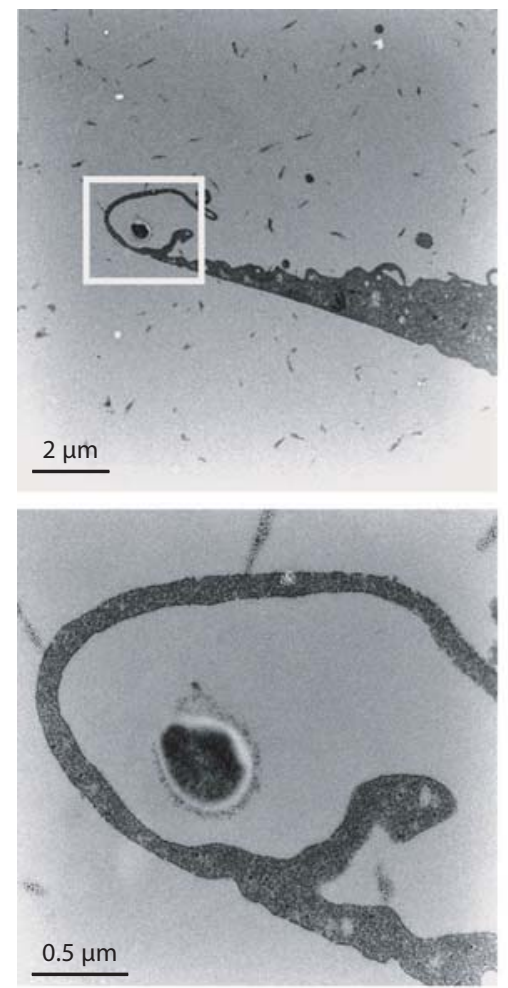

d b
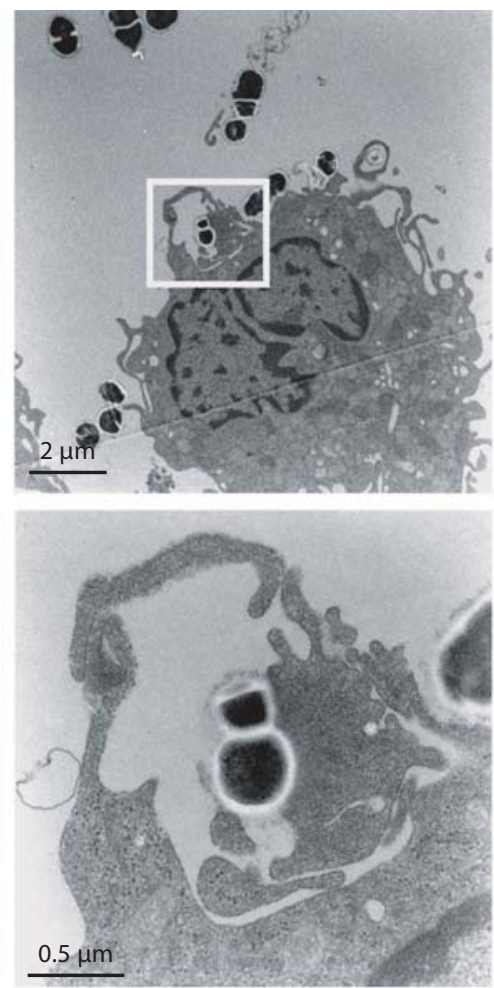

c
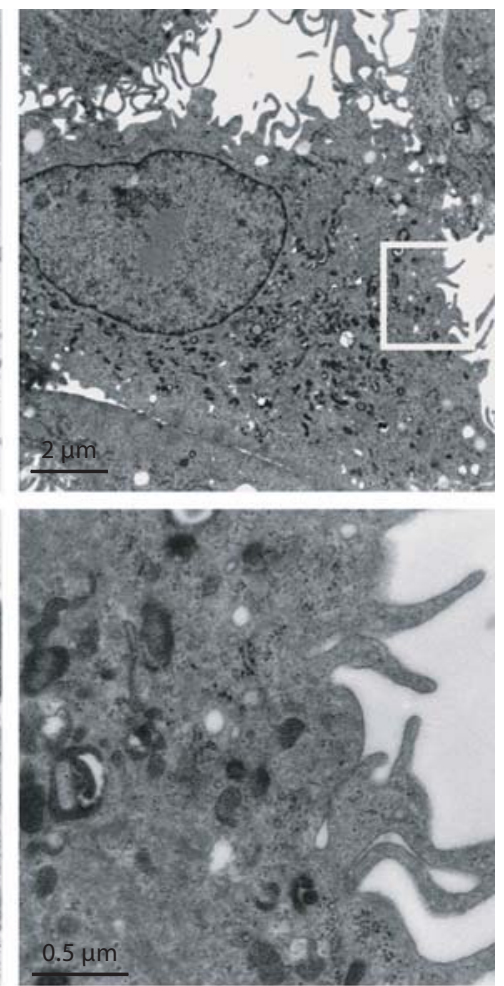

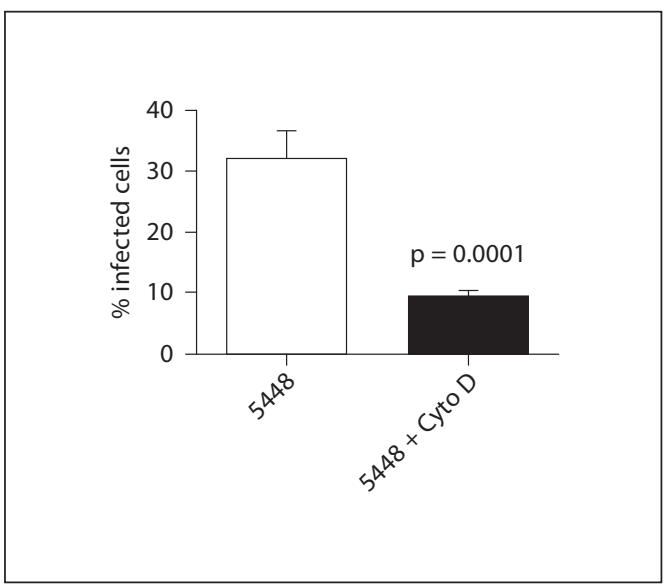

e

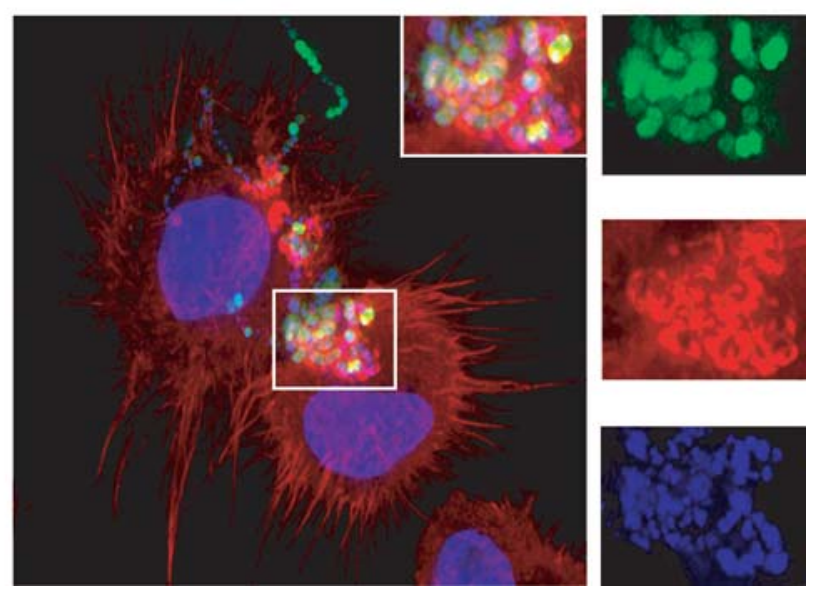

Fig. 1. Uptake of S. pyogenes in macrophages involves long asymmetrical pseudopod loops. Transmission electron microscopy analysis of hMDMs infected with wild-type 5448 after 10 min (a) and $1 \mathrm{~h}$ (b), or uninfected cells (c). Higher magnifications of the boxed areas are shown in the lower panels. Representative images of 1 experiment out of 3 performed using different donors are shown. d Percentage of cells harbouring GFP-expressing wildtype 5448 . Cells were pre-treated $+/-10 \mu \mathrm{g} / \mathrm{ml}$ cytochalasin D for 30 min followed by infection with GFP-expressing 5448 for $4 \mathrm{~h}$ and then analyzed by flow cytometry. The bars show mean \pm SEM of 9 observations using cells from 3 different donors. Statistically significant differences were determined by the MannWhitney test. e Representative fluorescent overlay image of wildtype 5448 uptake in macrophages at $1 \mathrm{~h}$ after infection with actin filaments visualized by Alexa Fluor phalloidin 555 (red), 5448 stained for the Lancefield group A carbohydrate (green) and cell nuclei DAPI (blue). Inset Higher magnification of the boxed area with each staining/channel shown separately. 
a
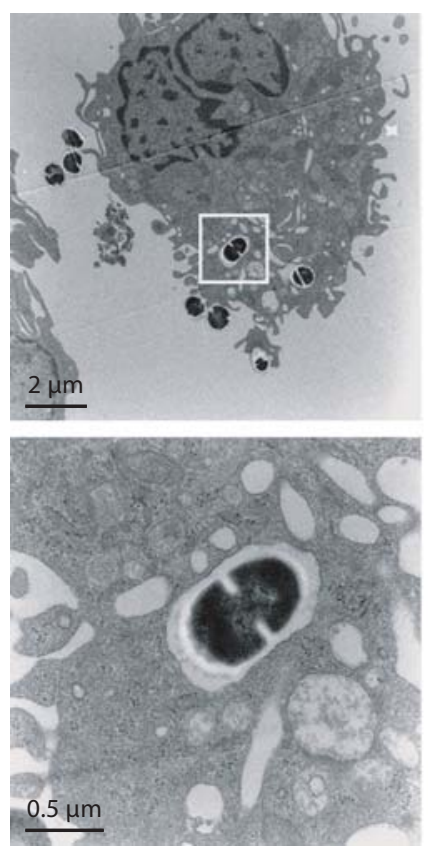

b
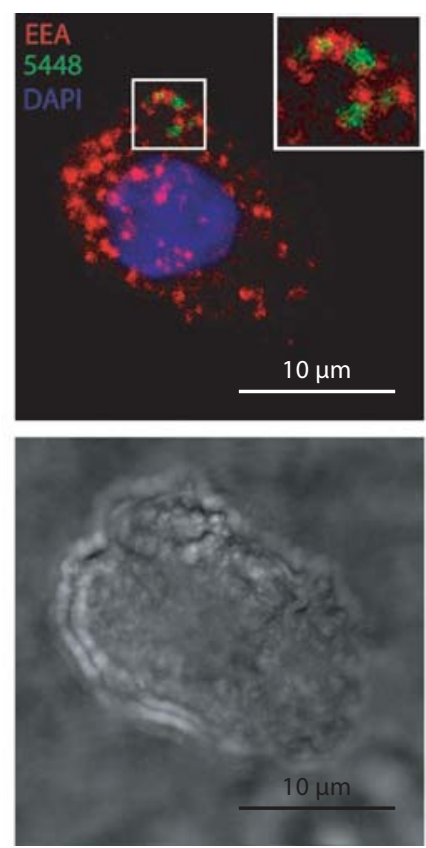

Fig. 2. Intracellular trafficking of $S$. pyogenes in macrophages. Transmission electron microscopy analyses revealed membranesurrounded $S$. pyogenes in infected hMDMs at $1 \mathrm{~h}$ after infection (a). Co-localization of GFP-expressing 5448 (green) with endosomal/lysosomal markers (red, Alexa 594) was assessed by immunofluorescent stainings and confocal analyses. Representative images of infected cells stained for early endosomal antigen 1

cells (data not shown). Four hours after infection, the frequency of cells harbouring viable bacteria was assessed by a bacterial viability stain. On average, $20-30 \%$ of the cells harboured live bacteria following infection with the wild-type 5448 strain, whereas significantly lower frequencies of cells contained viable $5448 \Delta \mathrm{M} 1$ bacteria $(\mathrm{p}=$ 0.03 ) (fig. 3a). Although the majority of intracellular 5448 wild-type bacteria were viable, it was also clear that a number of cells contained dead bacteria, likely resulting from an efficient phagocytic killing. We also performed experiments in which bacterial resurgence in the cell culture media was measured following removal of antibiotic after $4 \mathrm{~h}$ of infection. This demonstrated striking differences between the strains, as no viable bacteria were found in cell cultures infected with the mutant, as compared to $1 \times 10^{8} \mathrm{CFU}$ for wild-type 5448 (fig. 3b). To determine whether this finding resulted from a more efficient intracellular killing of the mutant or alternatively that the mutant was unable to egress out of the cells, infected cells were visualized by transmission electron mi- c
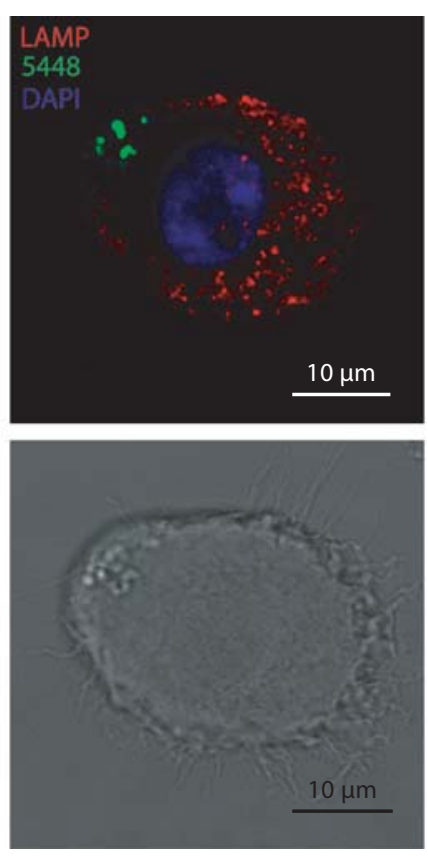

d
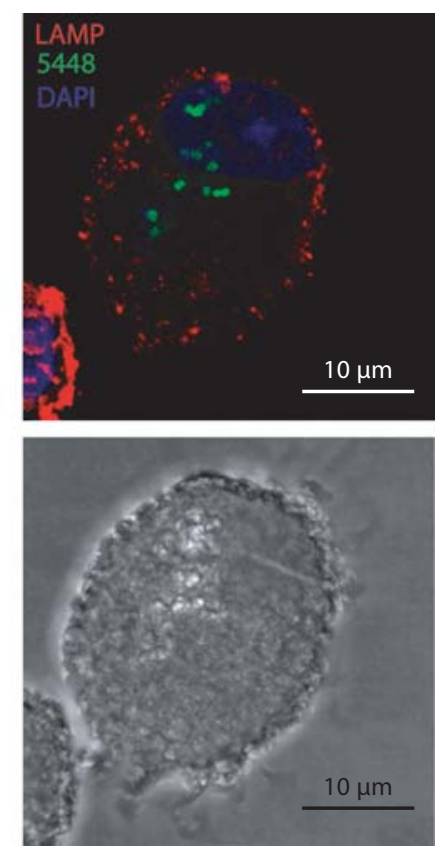

(EEA-1) at 5 min after infection (b) or for lysosomal membraneassociated protein 1 (LAMP-1) at $1 \mathrm{~h}$ (c) and $4 \mathrm{~h}$ (d) after infection. The inset figure shows the magnification of the boxed area (b). Higher magnifications of the boxed area, alternatively phase contrast images, are shown in the lower panel. All images are representatives of 3 separate experiments.

croscopy. At $6 \mathrm{~h}$ after infection, vesicle accumulation around, and fusion with, phagocytic vacuoles harbouring $5448 \Delta \mathrm{M} 1$, but not 5448 wild-type bacteria, was observed (fig. 3c, d). Further support for a differential fusion with lysosomal compartments was obtained through live-cell imaging of cells co-incubated with the lysosomal-specific stain LysoTracker and GFP-expressing 5448 strains (fig. 3e, f; online supplementary fig. 1, www. karger.com/doi/10.1159/000317635). Cells were monitored for up to $5 \mathrm{~h}$ after infection, and whereas only a minute proportion of the 5448 wild-type bacteria were found to co-localize with LysoTracker, the opposite was true for $5448 \Delta \mathrm{M} 1$ where bacterial vacuoles accumulated high amounts of this lysosomal-specific marker evident already $3 \mathrm{~h}$ after infection (online suppl. fig. 1).

\section{M1 Protein-Dependent Macrophage Modulation Is}

Associated with a Skewed Inflammatory Response

We further investigated the inflammatory state of infected macrophages and found important differences be- 


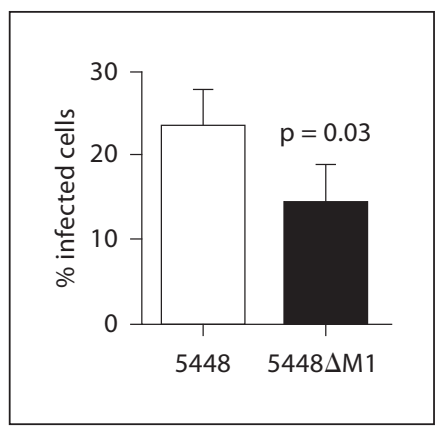

c
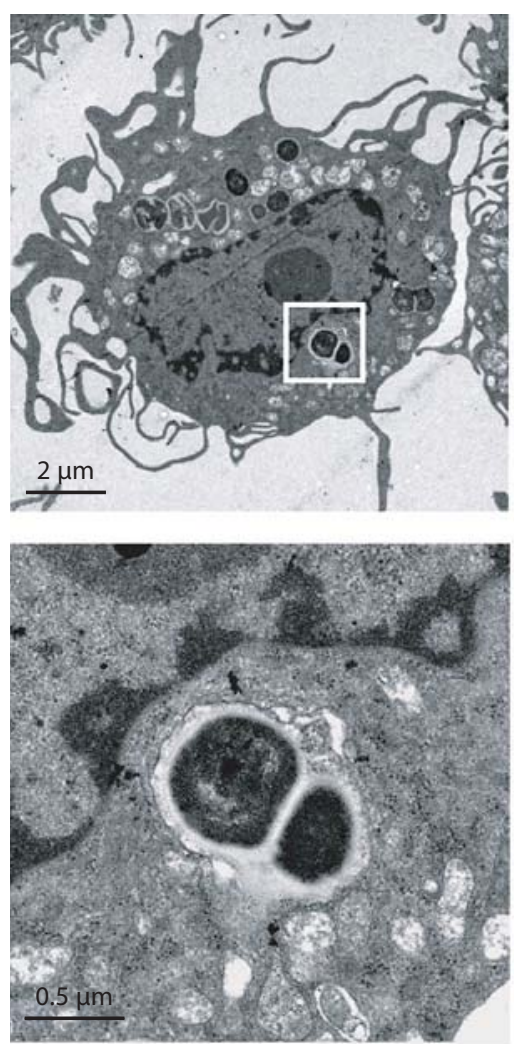

e
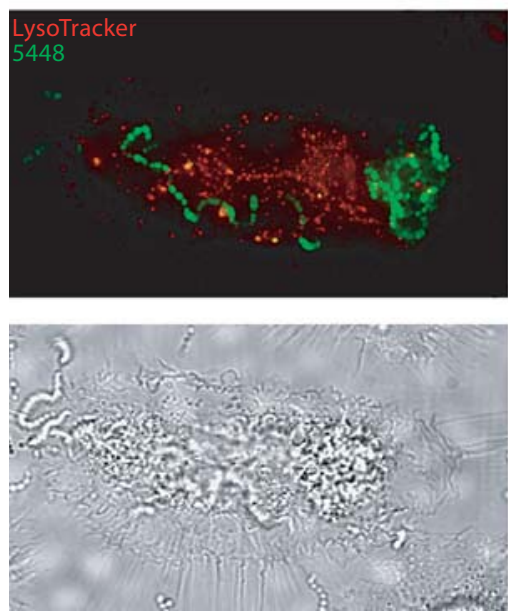

b

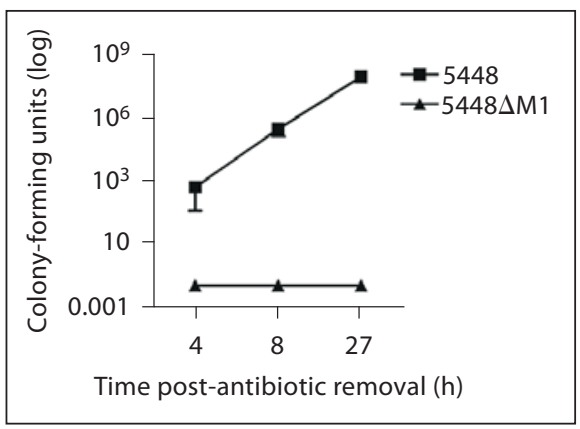

d
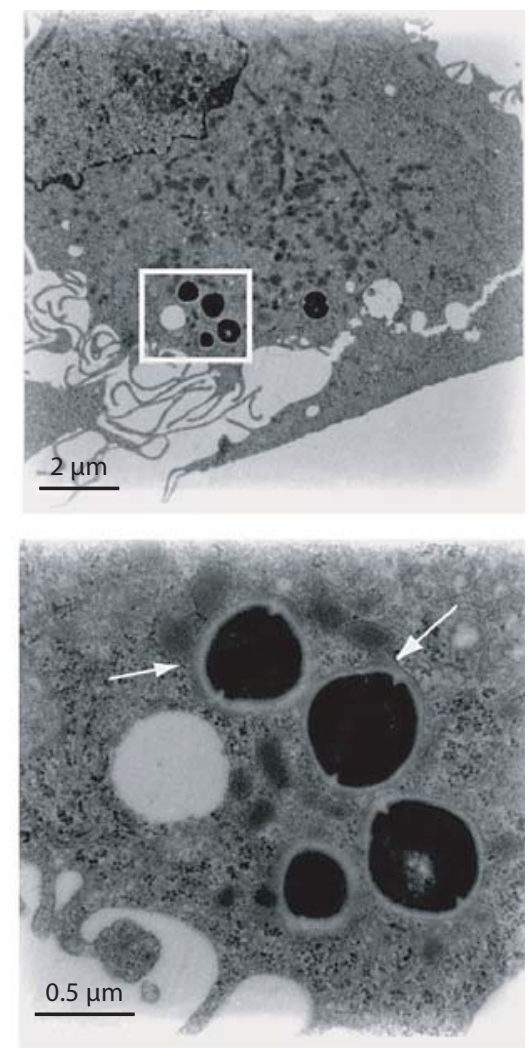

f
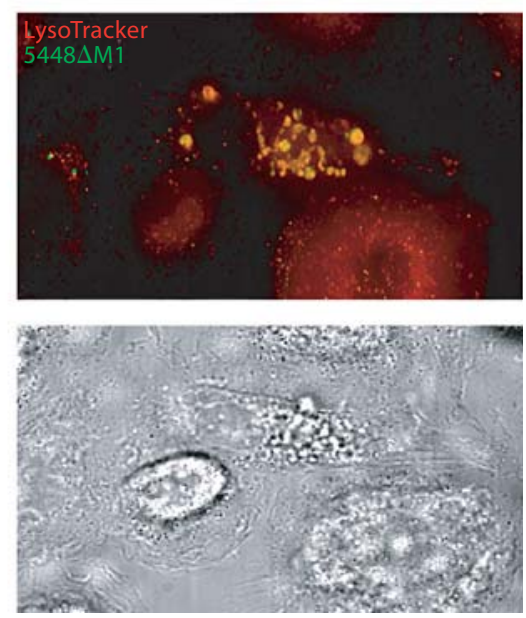


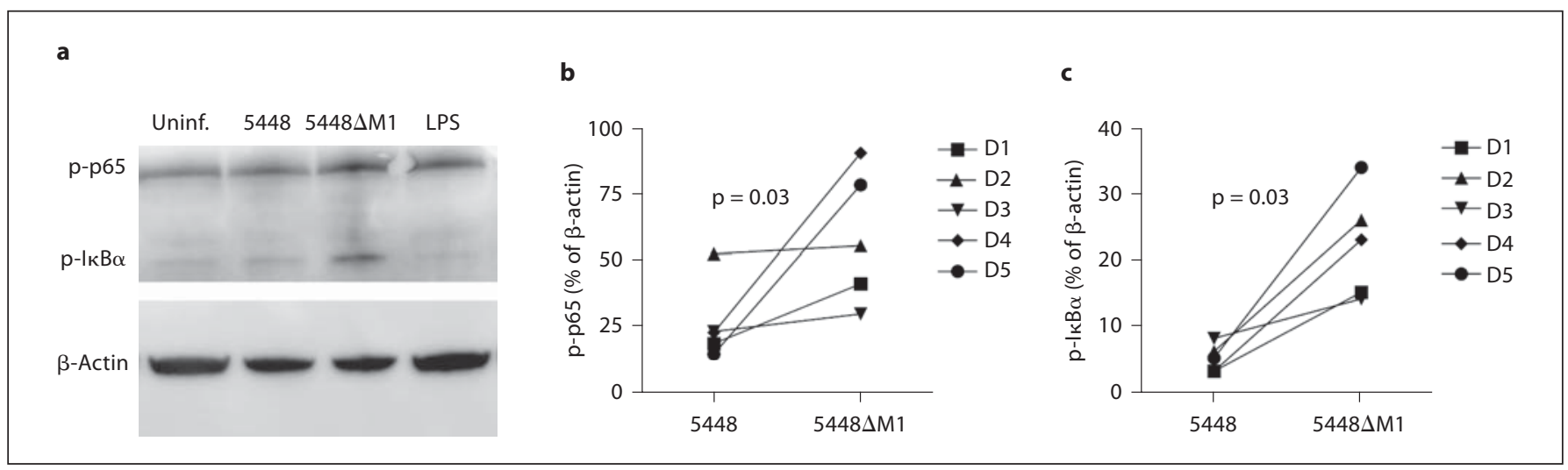

Fig. 4. M1 protein-dependent variation in NF- $\kappa \mathrm{B}$ signalling in infected macrophages. a Whole cell lysates from macrophages infected for $10 \mathrm{~min}$ with $S$. pyogenes 5448 or $5448 \Delta \mathrm{M} 1$. LPS-activated macrophages served as positive control. Proteins were transferred to polyvinylidene fluoride membranes that were stained with antibodies specific for $\mathrm{p}-\mathrm{I} \kappa \mathrm{B} \alpha, \mathrm{p}$-p65 or $\beta$-actin. Cell extracts from 5 different donors were tested, and the image shows 1 representative donor. The lack of phosphorylation of IкB $\alpha$ in
LPS-stimulated cells is due to a differential kinetics, inasmuch as a strong $\mathrm{p}$-ІК $\mathrm{B} \alpha$ was evident after $5 \mathrm{~min}$ of stimulation but not after $10 \mathrm{~min}$. The bars show phosphorylation of p-65(b) and IкB $\alpha$ (c) in relation to $\beta$-actin in cells infected with either 5448 or $5448 \Delta \mathrm{M} 1$ strains, quantified using ImageJ software. Each symbol indicates a separate donor, 5 donors in total. Statistically significant differences were determined by the Wilcoxon signed rank test. tween cells infected with 5448 wild type and $5448 \Delta \mathrm{M} 1$. First we analysed NF- $\kappa \mathrm{B}$ signalling responses in whole cell extracts of infected macrophages, which revealed a significantly higher level of phosphorylated p65 and phosphorylated I $\mathrm{B} \alpha$ following infection with $5448 \Delta \mathrm{M} 1$, as compared to the wild-type strain $(\mathrm{p}=0.03)$ (fig. 4). Based on the results recently published by Porta et al. [14], these data would translate into a suppressed inflammatory response in macrophages infected with $\mathrm{M} 1$ proteinexpressing $S$. pyogenes, as compared to the mutant. This is supported by preliminary experiments assessing TNF $\alpha$ gene expression $1 \mathrm{~h}$ after infection, which demonstrated an almost 5 -fold reduction in TNF $\alpha$ gene expression following infection with wild-type 5448 as compared to the M1-deficient mutant ( $\mathrm{p}=0.03$; based on gene expression data obtained from 6 different donors). Taken together, the data imply that M1 protein-dependent macrophage modulation involves suppression of the inflammatory signals that activate macrophage defences required for killing of intracellular pathogens.

\section{Intracellular Bacterial Replication, Egress and Re-Infection}

Ultrastructural data revealed that once inside its phagocytic vacuole, a significant proportion of $S$. pyogenes remained intact and showed no signs of degradation even at $12 \mathrm{~h}$ after infection (fig. 5a). In fact, the images indicated that there is an ongoing intracellular bacterial replication, as evident by cocci displaying the characteristic septum of dividing bacteria (fig. 5d). Further support for an intracellular replication was provided by the bacterial counts observed in cell cultures with antibiotic-free medium (fig. 3b), which were 10-fold higher than that obtained following growth of the same bacterial inoculum in culture medium without cells. The high extracellular bacterial counts also demonstrated the ability of the streptococci to leave their intracellular niche. This was further shown by electron micrographs that revealed bacteria egressing out of host cells already at $6 \mathrm{~h}$, although the most impressive release occurred at 8-12 h after infection (fig. 5a). In contrast, no bacterial egress could be noted in cells infected with fixed wild-type 5448 or $5448 \Delta \mathrm{M} 1$ (fig. 5b, c).

Another striking observation was the dramatic morphological changes that could be observed in wild-type 5448-infected cells. After $8 \mathrm{~h}$ of infection, the vacuoles containing bacteria appeared looser and more spacious than shortly after uptake (fig. $5 \mathrm{a}$ and $2 \mathrm{a}$, respectively), and at $12 \mathrm{~h}$ marked cell vacuolization and even cell burst was evident. Whether the cell burst is caused by the massive intracellular bacterial load or by enzymatic activity, such as that reported for streptolysin $\mathrm{O}[15,20-22]$, remains to be addressed. However, the data clearly demonstrated that viable bacteria were required in order to induce cell burst, as cells cultured with paraformaldehydefixed 5448 showed an intact healthy morphology despite 

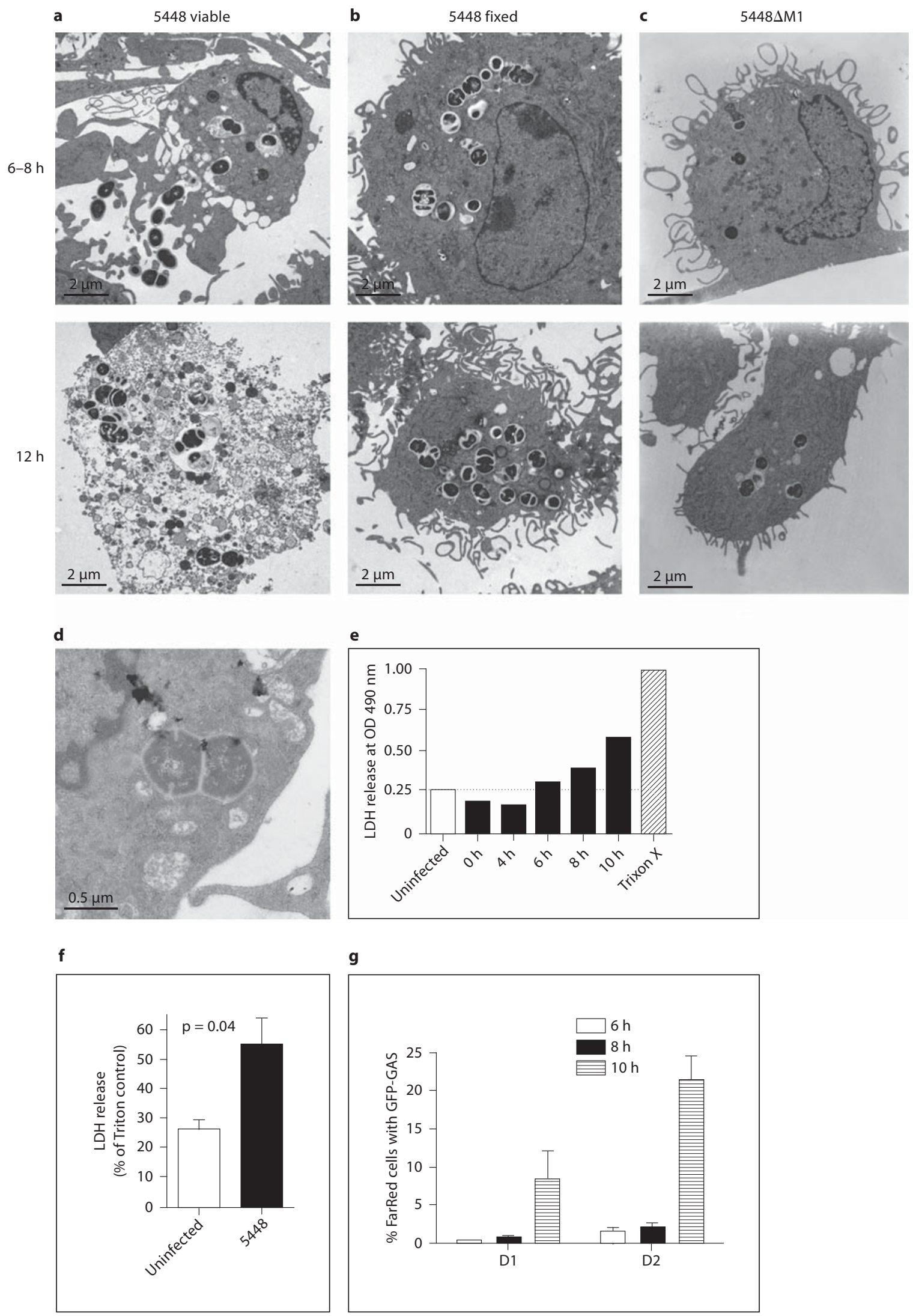
a heavy intracellular bacterial load (fig. 5b). A similar unaffected cellular morphology was noted in cells infected with $5448 \Delta \mathrm{M} 1$ (fig. $5 \mathrm{c}$ ), which indicates that it is not the infection per se but rather specific host-bacterial interactions that affect this phenomenon. Cell death was also monitored in kinetic experiments by measuring release of the cytosolic enzyme LDH in the cell culture media after infection with wild-type 5448. In agreement with microscopy data, a time-dependent significant increase in LDH release was noted in infected, as compared to uninfected, cells (fig. 5e, f).

Finally, we addressed the clinically relevant question whether the egressed bacteria were capable of re-infecting new cells in their surrounding environment. For this purpose, FarRed-labelled macrophages were added to already infected cells and infection of new cells was evaluated by flow-cytometrical analyses assessing GFP/FarRed double positivity. The data demonstrated that FarRedlabelled cells became infected with GFP streptococci (fig. $5 \mathrm{~g}$ ), thus providing evidence that intracellular bacteria may re-infect new cells.

\section{Discussion}

In this study, we demonstrate that $S$. pyogenes is capable of modulating the human macrophage to promote bacterial persistence within phagocytic vacuoles. A major factor contributing to this event is the M1 protein, which was noted to contribute to impaired fusion with lysosomal vesicle. The bacteria not only persist, but also replicate intracellularly, which results in a significant

Fig. 5. Intracellular replication is followed by bacterial egress and cell death. Transmission electron microscopy images of macrophages infected with viable (a) or paraformaldehyde-fixed (b) $S$. pyogenes wild-type 5448, or viable $5448 \Delta \mathrm{M} 1$ (c) for $6-8$ or $12 \mathrm{~h}$. d High-magnification electron micrograph of replicating bacteria showing the characteristic septum. e Cell death was quantified by calorimetrical measurement of LDH release from the cytosol of infected cells into the cell culture media at different time points after infection. $f$ Mean \pm SEM of LDH release at $10 \mathrm{~h}$ after infection of cells from 5 different donors. Statistically significant differences were determined by the paired t test. Ability of egressed bacteria to re-infect new cells was assessed by addition of FarRedlabelled cells to the cell cultures (for details see Material and Methods), and GFP-FarRed-double-positive cells were monitored by flow cytometry at different time points after infection. $\mathbf{g}$ Mean \pm SD of results obtained from 2 separate experiments using cells from different donors.

S. pyogenes in Macrophages bacterial reservoir within the cell. The study demonstrates for the first time that $S$. pyogenes can exploit and remodel the macrophage to promote persistence and continued infection.

Defining the ultrastructure of the uptake process is an important step towards understanding entry and intracellular trafficking of pathogens. To this end, we employed transmission electron microscopy to visualize $S$. pyogenes uptake in macrophages, which revealed membrane ruffles and pseudopods encircling the bacteria, eventually resulting in bacterial spacious vacuoles. Since no evidence of phagocytic cup formation or caveolae involvement was found, the uptake is likely achieved either through a form of coiling phagocytosis or triggered phagocytosis. In addition, ultrastructural data revealed large asymmetrical pseudopod loops reaching out and encircling the bacteria, a profile highly similar to the looping phagocytosis previously only described for Francisella tularensis uptake into macrophages [23]. Thus, this study demonstrates that in macrophages, $S$. pyogenes exploit uptake processes distinct from the previously described phagocytic uptake of $S$. pyogenes in neutrophils [9] and zipper-like mechanisms or caveolae-mediated endocytosis in non-phagocytic cells [24-26]. In macrophages, bacterial uptake did not seem to be dependent on a secreted factor, since both viable and paraformaldehyde-fixed bacteria behaved in an identical manner.

Once inside the host cell, the pathogens employ different strategies to avoid the phagosomal-lysosomal degradation pathway. Salmonella reside in spacious late endocytic compartments but avoid fusion with lysosomes [reviewed in 27]. In contrast, Brucella- and Legionellacontaining endosomes branch off early and convert into endoplasmic-reticulum organelles permissive of bacterial replication $[28,29]$. On the other hand, intracellular survival of Francisella involves prevention of phagosomal maturation, which eventually results in bacterial escape into the host cytosol [30,31]. In this paper, we show that $S$. pyogenes reside in phagocytic vacuoles that transiently associate with early endosomes, but do not acquire late endosomal or lysosomal markers, indicating an arrest in phagosome maturation. Importantly, lysosomal fusion with bacteria-containing phagocytic vacuoles that resulted in bacterial killing was evident when a $S$. pyogenes strain deficient in the M1 protein was used. This is in complete agreement with the previously reported M1 protein-dependent impairment in granule fusion in neutrophils $[9,19]$, and demonstrates a common immune evasion strategy employed by $S$. pyogenes to support intracellular persistence in phagocytic cells. Whether this

J Innate Immun 2010;2:534-545 
is a unique property to $\mathrm{M} 1$ protein or whether other $\mathrm{M}$ proteins may exert the same suppressive effect on macrophages and neutrophils is a clinically relevant question that warrants further studies. It is well known that M1 strains are overrepresented among severe invasive streptococcal infections, but other types may also give rise to these severe life-threatening infections and intracellular bacterial persistence in phagocytes has been demonstrated in patients infected with diverging $S$. pyogenes types [10].

Further insight into M1 protein modulation of macrophages was sought by exploration of host responses, in particular inflammatory responses. Macrophage polarization towards an anti-inflammatory phenotype (that is M2 phenotype) is a strategy shared by several pathogens to promote intracellular survival [reviewed in 13]. In line with this, a higher level of phosphorylation of p65 and I $\mathrm{B} \alpha$ was noted in cells infected with the M1 proteindeficient strain as compared to the wild-type strain. Thus, the results indicated that the inflammatory response was suppressed following infection with M1 protein-expressing $S$. pyogenes, a finding also supported by a reduction in TNF $\alpha$ gene expression. At first sight, these results may seem at odds with the well-described hyperinflammatory responses characterizing severe invasive streptococcal infections [32-35]. However, as bacteriamediated suppression of inflammation is likely to occur only at the single cell level, that is in cells harbouring viable bacteria, the overall response may well be pro-inflammatory. This is in agreement with a previous study, which demonstrated an atypical activation macrophage phenotype with both M1 (pro-inflammatory cytokines and chemokines) and M2 (IL-1ra, IL-10) markers following S. pyogenes infections in a murine model [36].

Another clinically important finding of this study is that of intracellular $S$. pyogenes replication in macrophages. In contrast, in non-phagocytic cells $S$. pyogenes survival over time is rather poor, a phenomenon attributed to autophagy $[22,37]$. In macrophages, $S$. pyogenes remain intact within phagocytic vacuoles for a prolonged time, and bacterial replication is evident from approximately $6 \mathrm{~h}$ after infection based on viable counts measurements and electron micrographs. Simultaneously, marked morphological changes of the bacteria-containing vacuoles become apparent. As the infection and bacterial replication proceeds, extensive vacuolization, cellular disruption and cell death occur which coincide with bacterial egress out of their host cells. Ultrastructural images reveal a seemingly organized process of bacterial egress out of the cell, which makes it tempting to specu- late that this precedes cell lysis rather than vice versa. The type of cell death and underlying mechanism is as yet unknown. Among the bacterial factors likely to be involved in this process, streptolysin $\mathrm{O}$ is a principal candidate as it is known to exert potent cytotoxic effects on a large variety of cells $[22,38]$, and has been implicated in different types of cell death, that is apoptosis and oncosis, of macrophages [21]. However, there are also other streptococcal factors with known cytotoxic and apoptotic activities, including among others streptolysin $\mathrm{S}$ and the cysteine protease SpeB, which may be involved in cell lysis $[38,39]$. It is noteworthy that in this study, S. pyogenesassociated cell lysis is not evident until later time points (8-12 $\mathrm{h}$ after infection), in contrast to the rapid cell death occurring within hours as reported previously $[20,21]$. One plausible explanation to this difference could be that while we based our studies on human primary monocyte-derived macrophages, the other reports focused on murine cells, a human monocytic cell line and/or human blood-derived monocytes. The fact that intracellular viable $S$. pyogenes can be detected in macrophages in infected tissue from patients for a prolonged time [10] supports our conclusion that bacterial modulation of the macrophage provides a hospitable niche for an extending time period during which bacterial replication occurs, resulting in a significant bacterial reservoir for continued infection and tissue injury.

The finding that M1 protein is involved in modulation of human macrophages adds yet another role to this multifunctional protein. Aside from its long-recognized anti-phagocytic activities in blood, it has recently been ascribed a central role in mediating shock and systemic toxicity associated with severe invasive streptococcal infections $[3,4,40]$. Taken together, this implicates M1 protein as a key factor and important target for novel therapeutic strategies in these severe infections.

\section{Acknowledgements}

We gratefully acknowledge Maria Baumgarten, Anette Hofmann, Julius Juarez and Lidija Bosnjak for excellent technical assistance. We thank Rita Wallén from the department of Cell and Organism Biology, Lund University, for providing the electron microscopy facilities. This work was supported in part by the foundations of Ragnar and Torsten Söderberg, Magn Bergvalls, Svenska sällskapet för medicinsk forskning, the Swedish Research Council (projects 16210 and 7480), Boehringer Ingelheim Fonds, Karolinska University Hospital and Karolinska Institutet. The funders had no role in study design, data collection and analysis, decision to publish or preparation of manuscript. 


\section{References}

1 Carapetis JR, Steer AC, Mulholland EK, Weber M: The global burden of group A streptococcal diseases. Lancet Infect Dis 2005;5: 685-694.

-2 Fraser JD, Proft T: The bacterial superantigen and superantigen-like proteins. Immunol Rev 2008;225:226-243.

- 3 Herwald H, Cramer H, Morgelin M, Russell W, Sollenberg U, Norrby-Teglund A, Flodgaard $\mathrm{H}$, Lindbom L, Bjorck L: M protein, a classical bacterial virulence determinant, forms complexes with fibrinogen that induce vascular leakage. Cell 2004;116:367-379.

-4 Pahlman LI, Morgelin M, Eckert J, Johansson L, Russell W, Riesbeck K, Soehnlein O, Lindbom L, Norrby-Teglund A, Schumann RR, Bjorck L, Herwald H: Streptococcal M protein: a multipotent and powerful inducer of inflammation. J Immunol 2006;177:1221-1228.

5 Kwinn LA, Nizet V: How group A Streptococcus circumvents host phagocyte defenses. Future Microbiol 2007;2:75-84.

-6 LaPenta D, Rubens C, Chi E, Cleary PP: Group A streptococci efficiently invade human respiratory epithelial cells. Proc Natl Acad Sci USA 1994;91:12115-12119.

7 Rohde M, Muller E, Chhatwal GS, Talay SR: Host cell caveolae act as an entry-port for group A streptococci. Cell Microbiol 2003;5: 323-342.

-8 Medina E, Rohde M, Chhatwal GS: Intracellular survival of Streptococcus pyogenes in polymorphonuclear cells results in increased bacterial virulence. Infect Immun 2003;71: 5376-5380.

-9 Staali L, Morgelin M, Bjorck L, Tapper H: Streptococcus pyogenes expressing $\mathrm{M}$ and $\mathrm{M}$ like surface proteins are phagocytosed but survive inside human neutrophils. Cell Microbiol 2003;5:253-265.

10 Thulin P, Johansson L, Low DE, Gan BS, Kotb M, McGeer A, Norrby-Teglund A: Viable group A streptococci in macrophages during acute soft tissue infection. PLoS Med 2006;3:e53.

11 Pizarro-Cerda J, Cossart P: Listeria monocytogenes membrane trafficking and lifestyle: the exception or the rule? Ann Rev Cell Develop Biol 2009;25:649-670.

-12 Santic M, Molmeret M, Klose KE, Abu Kwaik Y: Francisella tularensis travels a novel, twisted road within macrophages. Trends Microbiol 2006;14:37-44.

13 Benoit M, Desnues B, Mege JL: Macrophage polarization in bacterial infections. J Immunol 2008;181:3733-3739.

14 Porta C, Rimoldi M, Raes G, Brys L, Ghezzi P, Di Liberto D, Dieli F, Ghisletti S, Natoli G, De Baetselier P, Mantovani A, Sica A: Tolerance and M2 (alternative) macrophage polarization are related processes orchestrated by p50 nuclear factor kappaB. Proc Natl Acad Sci USA 2009;106:14978-14983.

15 Hakansson A, Bentley CC, Shakhnovic EA, Wessels MR: Cytolysin-dependent evasion of lysosomal killing. Proc Natl Acad Sci USA 2005;102:5192-5197.
16 Chatellier S, Ihendyane N, Kansal RG, Khambaty F, Basma H, Norrby-Teglund A, Low DE, McGeer A, Kotb M: Genetic relatedness and superantigen expression in group A streptococcus serotype M1 isolates from patients with severe and nonsevere invasive diseases. Infect Immun 2000;68:3523-3534.

17 Kadioglu A, Sharpe JA, Lazou I, Svanborg C, Ockleford C, Mitchell TJ, Andrew PW: Use of green fluorescent protein in visualisation of pneumococcal invasion of broncho-epithelial cells in vivo. FEMS Microbiol Lett 2001;194:105-110.

18 Kimoto H, Taketo A: Efficient electrotransformation system and gene targeting in pyogenic streptococci. Biosci Biotech Biochem 2003;67:2203-2209.

19 Staali L, Bauer S, Morgelin M, Bjorck L, Tapper H: Streptococcus pyogenes bacteria modulate membrane traffic in human neutrophils and selectively inhibit azurophilic granule fusion with phagosomes. Cell Microbiol 2006;8:690-703.

20 Goldmann O, Sastalla I, Wos-Oxley M, Rohde M, Medina E: Streptococcus pyogenes induces oncosis in macrophages through the activation of an inflammatory programmed cell death pathway. Cell Microbiol 2009;11: 138-155.

21 Timmer AM, Timmer JC, Pence MA, Hsu LC, Ghochani M, Frey TG, Karin M, Salvesen GS, Nizet V: Streptolysin O promotes group A Streptococcus immune evasion by accelerated macrophage apoptosis. J Biol Chem 2009;284:862-871.

22 Nakagawa I, Amano A, Mizushima N, Yamamoto A, Yamaguchi H, Kamimoto T, Nara A, Funao J, Nakata M, Tsuda K, Hamada S, Yoshimori T: Autophagy defends cells against invading group A Streptococcus. Science 2004;306:1037-1040.

23 Clemens DL, Lee BY, Horwitz MA: Francisella tularensis enters macrophages via a novel process involving pseudopod loops. Infect Immun 2005;73:5892-5902.

24 Dombek PE, Cue D, Sedgewick J, Lam H, Ruschkowski S, Finlay BB, Cleary PP: Highfrequency intracellular invasion of epithelial cells by serotype M1 group A streptococci: M1 protein-mediated invasion and cytoskeletal rearrangements. Mol Microbiol 1999;31: 859-870.

25 Talay SR, Zock A, Rohde M, Molinari G, Oggioni M, Pozzi G, Guzman CA, Chhatwal GS: Co-operative binding of human fibronectin to Sfbl protein triggers streptococcal invasion into respiratory epithelial cells. Cell Microbiol 2000;2:521-535.

26 Cue D, Dombek PE, Lam H, Cleary PP: Streptococcus pyogenes serotype M1 encodes multiple pathways for entry into human epithelial cells. Infect Immun 1998;66:4593-4601.

27 Bakowski MA, Braun V, Brumell JH: Salmonella-containing vacuoles: directing traffic and nesting to grow. Traffic 2008;9:20222031.
28 Shin S, Roy CR: Host cell processes that influence the intracellular survival of Legionella pneumophila. Cell Microbiol 2008;10: 1209-1220.

29 Celli J, de Chastellier C, Franchini DM, Pizarro-Cerda J, Moreno E, Gorvel JP: Brucella evades macrophage killing via VirB-dependent sustained interactions with the endoplasmic reticulum. J Exp Med 2003;198:545-556.

-30 Chong A, Wehrly TD, Nair V, Fischer ER, Barker JR, Klose KE, Celli J: The early phagosomal stage of Francisella tularensis determines optimal phagosomal escape and Francisella pathogenicity island protein expression. Infect Immun 2008;76:5488-5499.

- 31 Santic M, Asare R, Skrobonja I, Jones S, Abu Kwaik Y: Acquisition of the vacuolar ATPase proton pump and phagosome acidification are essential for escape of Francisella tularensis into the macrophage cytosol. Infect Immun 2008;76:2671-2677.

32 Norrby-Teglund A, Thulin P, Gan BS, Kotb M, McGeer A, Andersson J, Low DE: Evidence for superantigen involvement in severe group a streptococcal tissue infections. J Infect Dis 2001;184:853-860.

33 Norrby-Teglund A, Chatellier S, Low DE, McGeer A, Green K, Kotb M: Host variation in cytokine responses to superantigens determine the severity of invasive group A streptococcal infection. Eur J Immunol 2000;30:3247-3255.

34 Kotb M, Norrby-Teglund A, McGeer A, ElSherbini H, Dorak MT, Khurshid A, Green K, Peeples J, Wade J, Thomson G, Schwartz B, Low DE: An immunogenetic and molecular basis for differences in outcomes of invasive group A streptococcal infections. Nat Med 2002;8:1398-1404.

- 35 Sriskandan S, Unnikrishnan M, Krausz T, Dewchand H, Van Noorden S, Cohen J, Altmann DM: Enhanced susceptibility to superantigen-associated streptococcal sepsis in human leukocyte antigen-DQ transgenic mice. J Infect Dis 2001;184:166-173.

36 Goldmann O, von Kockritz-Blickwede M, Holtje C, Chhatwal GS, Geffers R, Medina E: Transcriptome analysis of murine macrophages in response to infection with Streptococcus pyogenes reveals an unusual activation program. Infect Immun 2007;75:4148-4157.

- 37 Joubert PE, Meiffren G, Gregoire IP, Pontini G, Richetta C, Flacher M, Azocar O, Vidalain PO, Vidal M, Lotteau V, Codogno P, Rabourdin-Combe C, Faure M: Autophagy induction by the pathogen receptor CD46. Cell Host Microbe 2009;6:354-366.

-38 Nizet V: Streptococcal beta-hemolysins: genetics and role in disease pathogenesis. Trends Microbiol 2002;10:575-580.

39 Nagamune H, Ohkura K, Ohkuni H: Molecular basis of group A streptococcal pyrogenic exotoxin B. J Infect Chemother 2005;11:1-8.

40 Pahlman LI, Olin AI, Darenberg J, Morgelin M, Kotb M, Herwald H, Norrby-Teglund A: Soluble M1 protein of Streptococcus pyogenes triggers potent $\mathrm{T}$ cell activation. Cell Microbiol 2008; 10:404-414. 